\title{
Association of SREBP2 gene polymorphisms with the risk of osteonecrosis of the femoral head relates to gene expression and lipid metabolism disorders
}

\author{
YANG SONG ${ }^{1,2}$, ZHENWU DU $^{1-3}$, BINGPENG CHEN $^{1,2}$, MING REN $^{1,2}$, QIWEI YANG $^{1-3}$, YUJIE SUI $^{3}$, \\ QINGYU WANG ${ }^{1,2}$, AO WANG $^{1,2}$, HAIYUE ZHAO $^{3}$, YANGUO QIN $^{1,2}$ and GUIZHEN ZHANG ${ }^{1-3}$ \\ ${ }^{1}$ Department of Orthopedics of the Second Hospital of Jilin University; ${ }^{2}$ Research Center of the Second Hospital \\ of Jilin University; ${ }^{3}$ The Engineering Research Center of Molecular Diagnosis and Cellular Treatment \\ for Metabolic Bone Diseases of Jilin Province, Changchun, Jilin 130041, P.R. China
}

Received August 1, 2016; Accepted July 12, 2017

DOI: $10.3892 / \mathrm{mmr} .2017 .7473$

\begin{abstract}
Although lipid metabolism disorders have been recognized as a primary factor of osteonecrosis of the femoral head $(\mathrm{ONFH})$, the molecular pathogenesis remains unclear. Sterol regulatory element-binding protein 2 (SREBP2) specifically regulates cholesterol and fatty acid metabolism to maintain lipid homeostasis. To explore the roles of the SREBP2 gene in the development of ONFH, the authors analyzed the gene polymorphism and gene expression of three tag single nucleotide polymorphisms of the SREBP2 gene, the serum lipids levels, and their associations with ONFH development in 182 ONFH patients and 179 healthy controls. The results demonstrated that the stage IV proportions of ONFH patients carrying the rs2267439CT or CC genotype were significantly higher and lower than the stage III proportions of ONFH patients $(\mathrm{P}=0.039)$, respectively. The serum triglyceride, low-density lipoprotein (LDL)-c levels, and LDL-C/high-density lipoprotein (HDL)-C ratio in the ONFH group were significantly increased compared to those in the control group $(\mathrm{P}=0.01, \mathrm{P}=0.005, \mathrm{P}=0.0001)$ while the HDL-C level of ONFH group was remarkably lower than that of the control group $(\mathrm{P}=0.0001)$. Association analysis further revealed that the LDL-c levels of the rs226744 GG and $\mathrm{AG}$ genotype carriers were statistically higher than that of the AA genotype carriers $(\mathrm{P}=0.039, \mathrm{P}=0.05)$. These results demonstrated that the gene polymorphism of SREBP2 not only significantly associated with the clinical phenotypes of
\end{abstract}

Correspondence to: Professor Yanguo Qin or Professor Guizhen Zhang, Department of Orthopedics of the Second Hospital of Jilin University, 218 Ziqiang Street, Nanguan, Changchun, Jilin 130041, P.R. China

E-mail: qinyangguo@hotmail.com

E-mail: zhangguizhenjlu@163.com

Key words: ONFH, SREBP2, gene polymorphism, gene expression, serum SREBP2 level, clinical phenotypes
ONFH but also closely related to lipid metabolism disorder. The results indicated that SREBP2 gene polymorphism and function may play key roles in the development of ONFH.

\section{Introduction}

Osteonecrosis of the femoral head (ONFH) is a complex disorder caused by interactions between genetic factors and environmental factors (1). It is characterized by a complicated pathological process, is difficult to diagnose in early stage, and eventually requires operation for artificial joint replacement. $\mathrm{ONFH}$ incidence has increased in recent decades. It is estimated that 20,000-30,000 new patients in the United States (2) and 150,000 to 200,000 new patients in China are diagnosed with ONFH annually, respectively. It is very important to elaborate the ONFN molecular pathogenesis and to identify its molecular targets to decrease the incidence. The polymorphisms of multiple genes have been suggested to be associated with ONFH risk. These polymorphisms are thought to cause disequilibrium with other genes, affecting ONFH occurrence, or to play a synergistic role in the development of ONFH by interfering with the expression of other genes (3-6) The ONFH patients often occur with a severe lipid metabolism disorder and the adipose accumulation in the impaired bone marrow cavity. Thus, lipid metabolism disorder has generally been regarded as a critical factor in ONFH pathogenesis, but the molecular mechanism of the adipose accumulation remains unclear.

Sterol regulatory element-binding protein 2 (SREBP2), a member of the SREBPs nuclear transcription factor family, binds to the sterol regulator, the promoter/enhancer in the lipid synthetic enzyme gene, to activate the target gene transcription and the genes expression of the cholesterol biosynthesis pathway, playing a key role in lipid homeostasis $(7,8)$. Considering the critical role of the SREBP2 gene in lipid homeostasis, the authors' research selected three Tag SNPs of the SREBP2 gene as molecular targets and investigated their genotypes, haplotypes, the serum SREBP2 expression and lipids level of ONFH patients, and their associations with the clinical phenotypes and the development of ONFH. 
Table I. Primers and probes of target SNPs of SREBP2 with LDR.

\begin{tabular}{|c|c|c|c|}
\hline dbSNP name & Primers $^{\mathrm{a}}$ & Probe $^{b}$ & Product size $^{c}$ \\
\hline $\begin{array}{l}\text { rs } 2267439 \\
{[C / T]}\end{array}$ & $\begin{array}{l}\text { Sense: AGCAGTTTTATTCAATTAG } \\
\text { AAC } \\
\text { Antisense:TGTTCAACAGGTACAC } \\
\text { AGGTG }\end{array}$ & $\begin{array}{l}\text { TC:TTTTTTTTAATTGTCCGTATCCTTCTGGA } \\
\text { TTCTC } \\
\text { TT:TTTTTTTTTTTAATTGTCCGTATCCTTCT } \\
\text { GGATTCTT } \\
\text { TR:P-GTCTGAACATTGCTTATCTGCGTACTT } \\
\text { TTTTTT-FAM- }\end{array}$ & $68 / \mathrm{C}, 71 / \mathrm{T}$ \\
\hline $\begin{array}{l}\text { rs1052717 } \\
{[\mathrm{A} / \mathrm{G}]}\end{array}$ & $\begin{array}{l}\text { Sense: TGAAGATTGGTTCAGCAA } \\
\text { TAGC } \\
\text { Antisense:CTCTGTAGCTTGGCAA } \\
\text { AGGCAC }\end{array}$ & $\begin{array}{l}\text { TA:TTTTTTTTTTTTTGAAGGTATTTATTGGG } \\
\text { CATCTTGTA } \\
\text { TG:TTTTTTTTTTTTTTTTGAAGGTATTTATT } \\
\text { GGGCATCTTGTG } \\
\text { TR:P-TTGATCACAGCACTGTGAATCCAGAT } \\
\text { TTTTTTTTTT-FAM- }\end{array}$ & $75 / \mathrm{A}, 78 / \mathrm{G}$ \\
\hline $\begin{array}{l}\text { rs } 2267443 \\
{[\mathrm{~A} / \mathrm{G}]}\end{array}$ & $\begin{array}{l}\text { Sense: AGTATCCAGTATCAACAC } \\
\text { TGGT } \\
\text { Antisense:ATAGCACTGTCAGAGT } \\
\text { GCCACT }\end{array}$ & $\begin{array}{l}\text { TA:AAGCTGCTTTGAGATTATGAGTA } \\
\text { TG:TATAAGCTGCTTTGAGATTATGAGTG } \\
\text { TR:P-TGTGTCTATAGAACAGGTTGTTT-FAM- }\end{array}$ & $47 / \mathrm{A}, 51 / \mathrm{G}$ \\
\hline
\end{tabular}

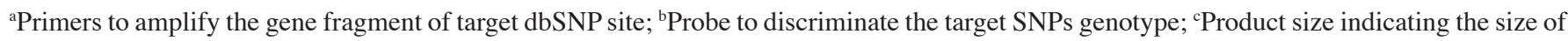
the product from a ligase reaction for each SNP genotype. SNP, single nucleotide polymorphism; SREBP2, sterol regulatory element-binding protein 2; LDR, ligase detection reaction.

\section{Materials and methods}

Patients and ethics statement. A total of 182 unrelated patients with ONFH (126 men, 56 women; age, $53.50 \pm 12.63$ years) were consecutively enrolled at the Department of Orthopedics of the Second Clinical College of Jilin University (Changchun, China) from March 2014 to June 2015. Patients with ONFH that was caused by direct trauma and patients with ONFH concurrent with cardiovascular diseases (arterial thrombosis and atherosclerosis etc.), congenital diseases, HIV infection, diabetes mellitus or renal dysfunction were excluded. ONFH diagnoses were established by evidence of osteonecrosis using plain radiographs in stages 2, 3 and 4 of the Ficat Classification system (9). According to the detailed disease history and etiological factors of the patients, the ONFH cases were classified into the following subgroups: alcohol-induced (68 cases, $38.42 \%$ ), idiopathic (67 cases, $37.85 \%$ ) and steroid-induced osteonecrosis (45 cases, 25.42\%). Steroid-induced osteonecrosis was defined by a history of taking a cumulative $2,000 \mathrm{mg}$ prednisolone or an equivalent over 21 days. Alcohol-induced osteonecrosis was defined by the consumption of $>900 \mathrm{ml}$ of pure ethanol per week. The disease course of ONFH ranged from 0.5 to 240 months, with an average of 72.45 months. There were 13 cases of stage II ONFH (7.34\%), 52 cases of stage III ONFH (29.38\%) and 112 cases of stage IV ONFH (63.28\%). There were six cases of ONFH patients who failed to undergo the clinical stages or aetiological classification duo to defect plain radiographs or unclear aetiological factors.

In addition, a total of 179 unrelated control subjects (114 men, 65 women; age, $52.58 \pm 11.08 \mathrm{yr}$.) that were ageand sex-matched with the ONFH group were consecutively enrolled at the Health Examination Center of the Second
Clinical College of Jilin University (Changchun, China) from October 2014 to December 2014. Control subjects were defined in the following way: They had no hip pain, their fasting serum blood glucose, triglyceride (TG) and total cholesterol (TC) levels were in the normal reference value range, abdominal ultrasound examination and chest X-ray radiography were normal, and they did not have a presence of cardiovascular or cerebrovascular diseases. All participants were of a Han Chinese population from northeast China.

The study was approved by the Ethics Committee of the Second Clinical College of Jilin University (Changchun, China), and conformed to the current ethical principles of the Declaration of Helsinki. All individuals provided written informed consent for their participation in the study. The authors declare that they have no competing financial interest.

Genomic DNA extraction and SNP selection. Genomic DNA was extracted from $2 \mathrm{ml}$ blood using a genomic DNA Extraction kit (DP318; Tiangen Biotech Co., Ltd., Beijing, China) following the manufacturer's protocols. The HapMap database and related literature were used to select tag SNPs in the SREBP2 gene, and the population distributions of the tag SNPs in different countries, nationalities and regions, especially in Asian populations, were analyzed. Three tag SNPs, rs2267439 [C/T], rs1052717 [A/G] and rs2267443 [A/G], in the intron region of the SREBP2 gene were selected as the research targets.

Genotyping. The detection primer, probe sequence and product size following ligase reaction of the $\mathrm{rs} 2267439$ [C/T], rs1052717 $[\mathrm{A} / \mathrm{G}]$ and rs2267443 [A/G] SNPs are presented in Table I.PCR reactions were performed in a buffer containing 
$11 \mathrm{DNA}, 1.5 \mu 1 \mathrm{MgCl}_{2}, 0.3 \mu 1 \mathrm{dNTPs}, 0.15 \mu \mathrm{l}$ primer mix, and $0.3 \mu \mathrm{l}$ Taq DNA ligase in a final reaction volume of $15 \mu \mathrm{l}$ (all reagents from Applied Biosystems; Thermo Fisher Scientific, Inc., Waltham, MA, USA). The reaction mixture was heated to $94^{\circ} \mathrm{C}$ for three min for denaturation. Then, the sample was subjected to 35 cycles of $94^{\circ} \mathrm{C}$ for $15 \mathrm{sec}$, annealing at $54^{\circ} \mathrm{C}$ for $15 \mathrm{sec}$ and extension at $72^{\circ} \mathrm{C}$ for $30 \mathrm{sec}$, followed by a final extension step at $72^{\circ} \mathrm{C}$ for $5 \mathrm{~min}$. The specific amplified fragments were used in ligase detection reaction (LDR) to identify the mutations associated with the rs2267439 [C/T], rs1052717 $[\mathrm{A} / \mathrm{G}]$ and $\mathrm{rs} 2267443[\mathrm{~A} / \mathrm{G}]$ SNPs.

The LDR assay was performed in a reaction volume of $10 \mu \mathrm{l}$ that contained $3 \mu \mathrm{l}$ PCR product, $1 \mu \mathrm{l} 10 \mathrm{X}$ ligase reaction buffer, $0.125 \mu \mathrm{l}(40 \mathrm{U} / \mu \mathrm{l})$ Taq DNA ligase and $0.01 \mu \mathrm{l}$ probe (10 p)/each probe; deionized $\mathrm{H}_{2} \mathrm{O}$ was added to a final volume of $10 \mu \mathrm{l}$. The ligation reaction was performed using a GeneAmp PCR System 9700 (Applied Biosystems Inc.; Thermo Fisher Scientific, Inc.) with the following temperature program: $2 \mathrm{~min}$ at $95^{\circ} \mathrm{C}$ and 30 cycles of $30 \mathrm{sec}$ at $94^{\circ} \mathrm{C}$ and $3 \mathrm{~min}$ at $56^{\circ} \mathrm{C}$. The products were analyzed using an ABI PRISM 3730xl DNA sequencer (Applied Biosystems Inc.; Thermo Fisher Scientific, Inc.). Chromas software version 2.6.4 (Technelysium Pty Ltd., South Brisbane, Australia) was used to analyze the sequencing peak chart.

Serum SREBP2 protein expression. Serum SOX9 protein expression was detected using an ELISA kit (DRE12665; Beijing Solarbio Science \& Technology Co., Ltd., Beijing, China) according to the manufacturer's protocols. Briefly, the assay range of SREBP2 was 5-300 pg/ml. The SREBP2 densities of standards wells on the ELISA plates were 300, $200,100,50$ and $25 \mathrm{pg} / \mathrm{ml}$, respectively. Blank wells [without sample or horseradish peroxidase (HRP)-conjugate reagent] were kept separately. Then, $40 \mu 1$ sample dilution and $10 \mu \mathrm{l}$ serum were added to the testing sample wells $40 \mu 1$ sample dilution and $10 \mu 1$ serum (final dilution of sample, 5-fold). These was incubated for $30 \mathrm{~min}$ at $37^{\circ} \mathrm{C}$, before being washed with PBS five times. The HRP-conjugate reagent $(50 \mu 1)$ was added and was incubated for $30 \mathrm{~min}$ at $37^{\circ} \mathrm{C}$. Following this, it was washed five times, and Chromogen Solutions A and B (50 $\mu 1$ each) were added separately and kept in a dark place for $15 \mathrm{~min}$ at $37^{\circ} \mathrm{C}$. The Stop Solution $(50 \mu \mathrm{l})$ was added to each well. Within $15 \mathrm{~min}$, the blank well was taken as zero, and absorbance was read at $450 \mathrm{~nm}$ using a multi-wavelength microplate reader (Varioskan; Thermo Fisher Scientific, Inc.).

Statistical analysis. Shesis software platform (http://analysis.bio-X.cn/SHEsisMain.htm) was used to analyze the Hardy-Weinberg equilibrium and haplotypes between the $\mathrm{ONFH}$ and control groups. Logistical regression analyses were used to calculated the odds ratios (OR), 95\% confidence intervals (CI), and corresponding p-values of each SNP, controlling for age and sex as covariates. The genetic models of dominant, recessive, and codominant were considered, and the genotypes were given codes of 0,1 , and 2; 0,1 , and 1 ; or 0,0 , and 1 in the codominant, dominant and recessive models, respectively. SPSS software (version, 10.0; SPSS, Inc., Chicago, IL, USA) was used to analyze the differences in serum SOX9 protein expression between the ONFH and control groups and the

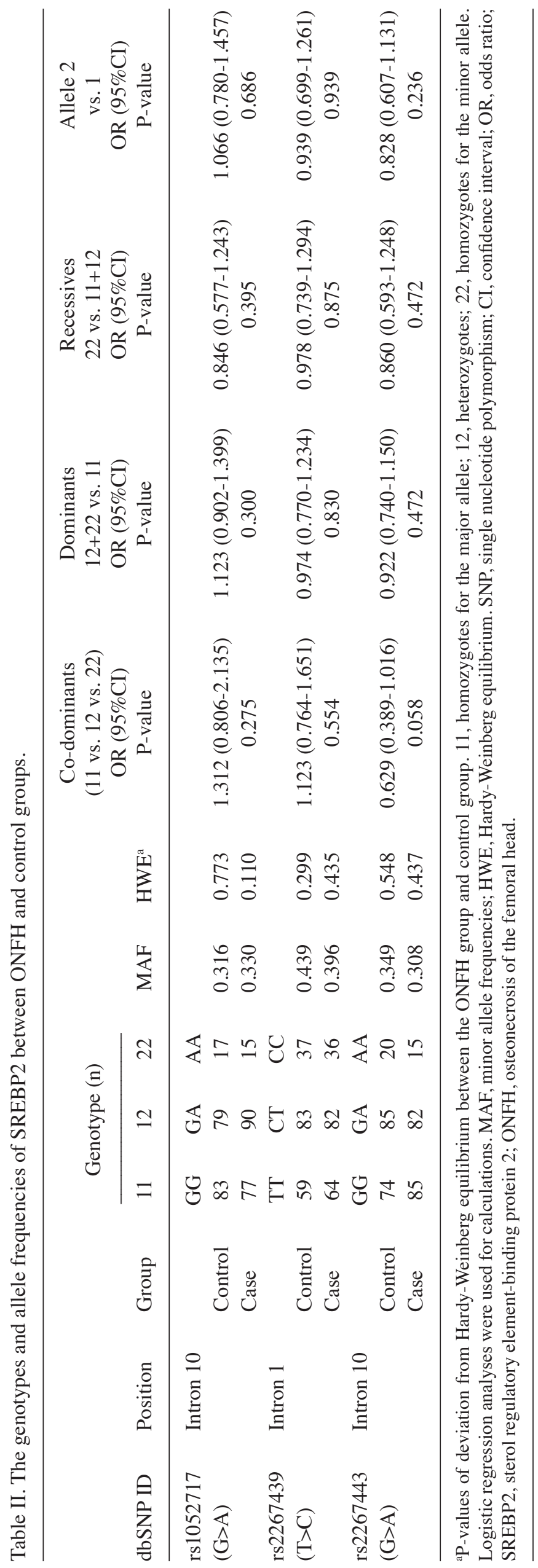


Table III. Haplotype frequencies of SREBP2 SNPs between ONFH and control groups.

\begin{tabular}{lcccccr}
\hline \multirow{2}{*}{ Gene dpSNPs } & \multicolumn{5}{c}{ Frequency } \\
\cline { 3 - 4 } & \multicolumn{2}{c}{ Haplotype } & Controls, n (\%) & Patients, n (\%) & OR (95\%CI) & P-value $^{\mathrm{a}}$ \\
\hline & Hap1 & A-T-A & $93.86(26.2)$ & $90.83(25.0)$ & $0.936(0.670-1.307)$ & 0.697 \\
SREBP2 & Hap2 & A-T-G & $16.64(4.6)$ & $29.16(8.0)$ & $1.787(0.961-3.324)$ & 0.063 \\
rs1052717 (A/G) & Hap3 & G-C-G & $153.22(42.8)$ & $150.08(41.2)$ & $0.938(0.698-1.260)$ & 0.670 \\
rs2267439 (C/T) & Hap4 & G-T-A & $28.45(7.9)$ & $17.25(4.7)$ & $0.576(0.311-1.068)$ & 0.076 \\
rs2267443 (A/G) & Hap5 & G-T-G & $62.06(17.3)$ & $72.76(20.0)$ & $1.191(0.819-1.734)$ & 0.358 \\
& Hap6 & G-C-A & $1.28(0.4)$ & $3.91(1.1)$ & - & - \\
& Hap7 & A-C-A & $1.41(0.04)$ & $0.01(0.00)$ & - & - \\
& Hap8 & A-C-G & $1.09(0.03)$ & $0.00(0.00)$ & - & - \\
\hline
\end{tabular}

SREBP2, sterol regulatory element-binding protein 2; SNPs, single nucleotide polymorphisms; ONFH, osteonecrosis of the femoral head; OR, odds ratio; CI, confidence interval. ${ }^{a} \chi^{2}$ test. Those with a frequency $<0.03$ were not analyzed.

associations of protein expression with genotypes and clinical phenotypes using the Student's t-test, ANOVA and $\chi^{2}$ test, respectively. Shesis software (haplotype analysis) was also used to analyze the correlation of the haplotype with the clinical phenotypes. Data were presented as mean \pm standard deviation and $\mathrm{P}<0.05$ was considered to indicate a statistically significant difference.

\section{Results}

The genotypes, allele frequencies and haplotypes of rs1052717 (A/G), rs2267439 (C/T) and rs2267443 (A/G) of the SREBP2 gene. SREBP2 genotypes and allele frequencies are presented in Table II. Logistical regression analysis indicated that although possessing no statistical significance, the co-dominant model of rs2267443 exhibited a higher tendency $(\mathrm{P}=0.058)$. There are eight haplotypes (A-T-A, A-T-G, G-C-G, G-T-A, G-T-G, G-C-A, A-C-A and A-C-G) among rs1052717, rs2267439 and rs2267443. Although the haplotypes between $\mathrm{ONFH}$ and control groups failed to show a statistical difference, the frequencies of the A-T-G (8.0\%) and G-T-A (4.7\%) haplotypes in the ONFH group were higher and lower tendency, respectively, than those of the control group (4.6, $7.9 \%$; $\mathrm{P}=0.063, \mathrm{P}=0.076$ ), as presented in Table III. The other three haplotypes were not statistically analyzed due to low population frequencies.

Associations of the genotypes and haplotypes of rs 1052717, rs2267439 and rs2267443 with the clinical phenotypes of ONFH. Correlation analysis of the SREBP2 genotypes with the disease course, etiological classification, unilateral or bilateral hip lesions, and clinical staging of $\mathrm{ONFH}$ demonstrated that the rs2267439 genotypes demonstrated a significant association with the clinical stages of ONFH. The proportions of stage IV patients carrying the rs2267439CT $(50.9 \%)$ or CC (13.4\%) genotype were significantly higher and lower, respectively, compared with those of stage III patients $(32.7,26.9 \%, \mathrm{P}=0.039)$, as indicated in Table IV. The correlation analysis between the SREBP2 haplotypes and the clinical stages or the unilateral and bilateral hip lesions of ONFH patients showed no significant differences, as presented in Tables $\mathrm{V}$ and VI.

Serum levels of TC, TG, high-density lipoprotein (HDL)-c, low-density lipoprotein $(L D L)-c, L D L-C / H D L-C$ ratios and their association with SREBP2 gene polymorphism. The serum TG, LDL-C levels and LDL-C/HDL-C ratios of the ONFH group were significantl $\quad \mathrm{y}$ higher than those of the control group $(\mathrm{P}=0.01, \mathrm{P}=0.005$ and $\mathrm{P}=0.0001$ respectively), while the HDL-C levels in the ONFH group was statistically lower, compared with that of the control group $(\mathrm{P}=0.0001)$, as presented in Fig. 1. The correlation analysis between the rs1052717, rs226743 and, rs2267443 genotypes and serum lipid levels indicated that the serum LDL-C levels of ONFH patients carrying the rs 2267443 GG or AG genotypes were significantly increased than those of patients carrying the AA genotype $(\mathrm{P}=0.039, \mathrm{P}=0.05)$, as presented in Fig. 1 .

Serum protein expression of SREBP2 gene and its association with SREBP2 genotypes and the clinical phenotypes of $O N F H$. ELISA analysis revealed that serum SREBP2 protein expression was not significantly different between the ONFH and control groups. The correlation analysis between the genotypes and serum SREBP2 protein expression presented no statistical significance, as demonstrated in Fig. 2. The association analysis of the serum SREBP2 protein level with the etiological classification, clinical stages and the unilateral or bilateral hip lesions of ONFH patients also presented no significant differences, as displayed in Fig. 3.

\section{Discussion}

SREBPs are transcription activation factors that regulate endogenous cholesterol and fatty acid synthesis and are expressed in all tissues and organs of that exhibit lipid synthesis $(10,11)$. A previous study demonstrated that $\mathrm{OA}$ is a metabolic disease involving the genetic deregulation of lipid and cholesterol metabolism. A genetic association analysis demonstrated a significant association between the $1784 \mathrm{G}>\mathrm{C}$ mutation of the SREBP2 gene and OA development in a 


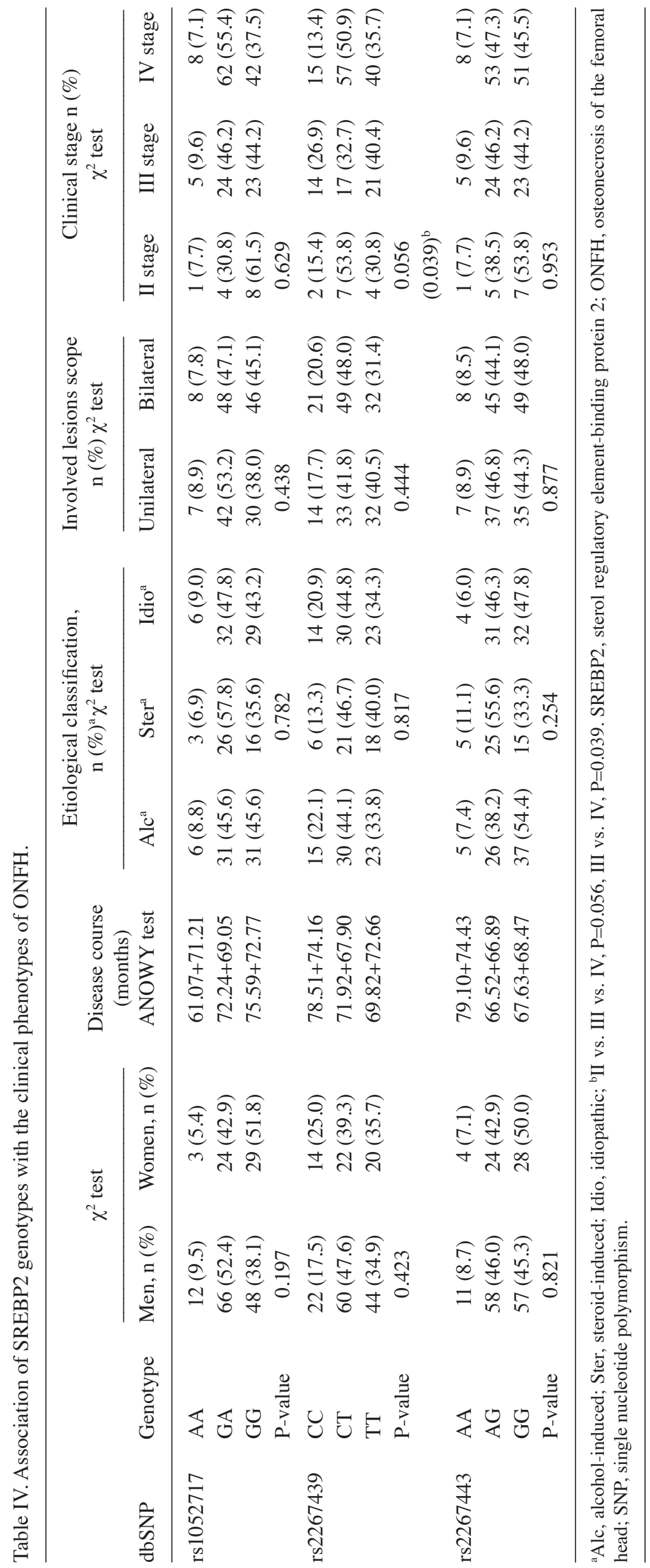


Table V. The association of SREBP2 haplotypes with the unilateral and bilateral hips lesions of ONFH.

\begin{tabular}{lccccc}
\hline Haplotypes & Unilateral, $\mathrm{n}(\%)$ & Bilateral, $\mathrm{n}(\%)$ & $\chi^{2}$ & P-value & OR (95\% CI) \\
\hline \multirow{2}{*}{ SREBP2 } & $40.58(25.7)$ & $50.15(24.3)$ & 0.081 & 0.775 & $0.933(0.577-1.506)$ \\
& $15.41(9.8)$ & $13.84(6.7)$ & 1.106 & 0.293 & $0.667(0.313-1.424)$ \\
$\mathrm{rs} 1052717(\mathrm{~A} / \mathrm{G})$ & $58.63(37.1)$ & $91.60(44.5)$ & 1.556 & 0.212 & $1.312(0.856-2.010)$ \\
$\mathrm{rs} 2267439(\mathrm{C} / \mathrm{T})$ & $8.05(5.1)$ & $9.45(4.6)$ & 0.050 & 0.823 & $0.896(0.342-2.351)$ \\
$\mathrm{rs} 2267443(\mathrm{~A} / \mathrm{G})$ & $32.95(20.9)$ & $39.56(19.2)$ & 0.147 & 0.701 & $0.904(0.538-1.517)$ \\
& $2.37(1.5)$ & $1.41(0.7)$ & - & - & - \\
& $0.00(0.0)$ & $0.01(0.0)$ & - & - & - \\
& $1.09(0.03)$ & $0.00(0.00)$ & & & \\
\hline
\end{tabular}

Those with a frequency $<0.03$ were not analyzed. SREBP2, sterol regulatory element-binding protein 2; ONFH, osteonecrosis of the femoral head; OR, odds ratio; CI, confidence interval.

Table VI. The association of SREBP2 haplotypes with clinical stages of ONFH patients.

\begin{tabular}{|c|c|c|c|c|c|c|}
\hline \multicolumn{2}{|c|}{ Haplotypes } & \multirow{2}{*}{$\begin{array}{c}\text { Stage IV, n (\%) } \\
56.54(25.2)\end{array}$} & \multirow{2}{*}{$\begin{array}{c}\text { Stage III, n (\%) } \\
27.30(26.3)\end{array}$} & \multirow{2}{*}{$\frac{\chi^{2}}{0.037}$} & \multirow{2}{*}{$\frac{\text { P-value }}{0.846}$} & \multirow{2}{*}{$\begin{array}{c}\text { OR }(95 \% \mathrm{CI}) \\
0.949(0.557-1.615)\end{array}$} \\
\hline SREBP2 & A-T-A & & & & & \\
\hline & A-T-G & $21.45(9.6)$ & $6.70(6.4)$ & 0.895 & 0.344 & $1.541(0.626-3.793)$ \\
\hline rs105271(A/G) & G-CG & $84.22(37.6)$ & $43.76(42.1)$ & 0.598 & 0.439 & $0.829(0.515-1.334)$ \\
\hline rs2267439(C/T) & G-T-A & $9.68(4.3)$ & $5.46(5.2)$ & 0.137 & 0.711 & $0.816(0.278-2.394)$ \\
\hline \multirow[t]{4}{*}{ rs2267443(A/G) } & G-T-G & $49.32(22.0)$ & $19.54(18.8)$ & 0.451 & 0.502 & $1.222(0.680-2.195)$ \\
\hline & G-C-A & $2.77(1.2)$ & $1.24(1.2)$ & - & - & - \\
\hline & A-C-A & $0.01(0.0)$ & $0.00(0.0)$ & - & - & - \\
\hline & A-C-G & $1.09(0.03)$ & $0.00(0.00)$ & - & - & - \\
\hline
\end{tabular}

All those with a frequency $<0.03$ were not analyzed. SREBP2, sterol regulatory element-binding protein 2; ONFH, osteonecrosis of the femoral head; OR, odds ratio; CI, confidence interval.

cohort of 1,410 Greek OA patients and healthy controls (12). A study in a Chinese Han population also demonstrated that the SREBP-2 rs2228314 polymorphism was closely associated with increased risk of knee OA (13).

Few reports have shown an association between SREBP-2 polymorphism and ONFH risk. In 2008, Kim et al (14) reported the protective effect of the SREBP-2 rs2267439 C allele against ONFH risk and the significant associations of rs1052717 and rs2267443 with increased ONFH risk. In 2012, the authors confirmed that the rs2267439TT and rs2267443GA genotype of the SREBP2 gene were associated with increased ONFH risk, while rs $2267439 \mathrm{C}$ allele frequency was associated with decreased ONFH risk (15). Lipid metabolism disorder has been recognized as a main factor in ONFH pathogenesis. The results of clinical and animal experiments have demonstrated that steroid- and alcohol-induced ONFH are associated with lipid metabolism disorder $(16,17)$. Considering both the critical effects of SREBP2 on lipid homeostasis and the role of lipid metabolism disorder in the development of ONFH, the authors conducted the genotype and haplotype analyses of rs1052717, rs2267439 and rs2267443 of the SREBP2 gene in $182 \mathrm{ONFH}$ patients and 179 controls. The results revealed that, although no statistical significance, the A-T-G haplotype frequency of the ONFH group was lower tendency while the
G-T-A haplotype frequency of the ONFH group was higher tendency than that of the control group, indicating that the A-T-G haplotype may be associated with a lower risk of ONFH and the G-T-A haplotype might be associated with a higher risk of ONFH. In the expanded sample system, the roles of A-T-G and G-T-A haplotypes in the development of ONFH remain to be deeply investigated.

To further explore the association of SREBP2 polymorphism with ONFH risk, conducted correlation analyses were conducted between the genotypes and haplotypes of the SREBP2 gene and the clinical phenotypes of ONFH. The results demonstrated that the SREBP2 rs2267439 genotypes were significantly associated with the clinical stages of ONFH; the frequencies of stage IV patients carrying the homozygous CC genotype and heterozygous CT genotype of rs 2267439 were significantly lower and higher, respectively, than those of stage III patients, suggesting that the CC genotype may be a protective genotype against ONFH hip lesion development, while the CT genotype may indicate a higher risk of ONFH hip lesion development. To investigate the effects of SREBP2 polymorphisms on gene expression, the authors examined serum SREBP2 protein expression and no significant difference was identified between the ONFH and control groups. As a key transcription factor, SREBP2 gene expression is 
A

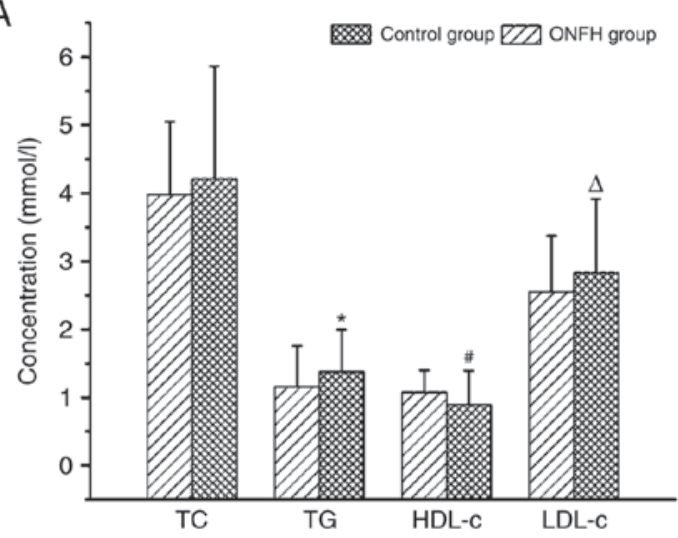

B 7 T TC TG HDL.C $\boxminus$ TLLLC

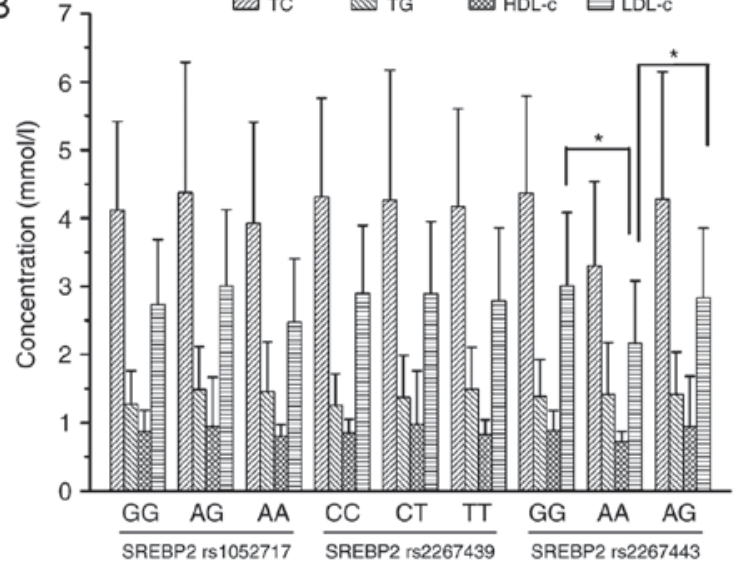

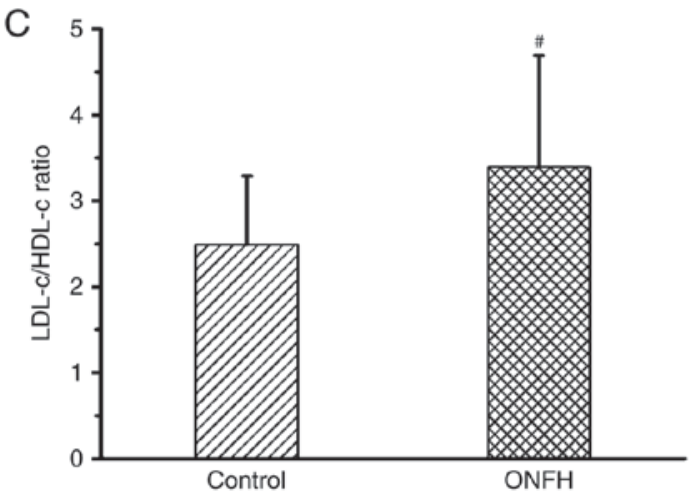

Figure 1. The serum lipid levels between the ONFH and control groups, and the association of SREBP2 genotypes with the serum lipid levels of ONFH patients. (A) The serum levels of TC, TG, HDL-c, and, LDL-c between the ONFH and control groups. ${ }^{~} \mathrm{P}<0.01,{ }^{\Delta} \mathrm{P}<0.005$, ${ }^{*} \mathrm{P}<0.0001$ vs. control. (B) The association of SREBP2 genotypes with the serum lipid levels of the ONFH group. ${ }^{*} \mathrm{P}<0.05$ as indicated. (C) The LDL-c/HDL-c ratio between the ONFH and control groups. ${ }^{~} \mathrm{P}<0.0001$ vs. control. Data are presented as mean \pm standard deviation. ONFH, osteonecrosis of the femoral head; SREBP2, sterol regulatory element-binding protein 2; TC, total cholesterol; TG, triglyceride; HDL, high-density lipoprotein; LDL, low-density lipoprotein.
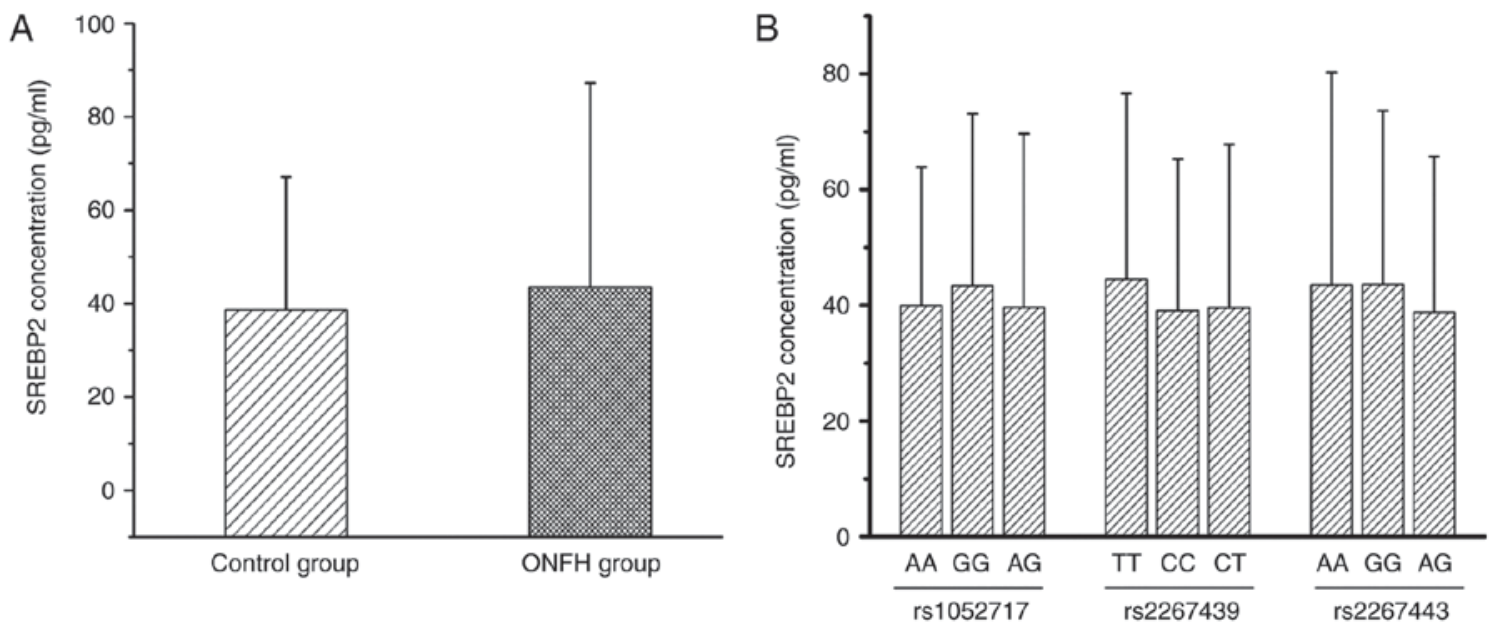

Figure 2. Serum SREBP2 protein expression and the association of protein expression with SREBP2 genotypes in ONFH patients. (A) The serum protein expression of SREBP2 gene between the ONFH and control groups. (B) The association of SREBP2 genotypes with protein expression in ONFH patients. Data are presented as mean \pm standard deviation. SREBP2, sterol regulatory element-binding protein 2; ONFH, osteonecrosis of the femoral head.

affected by multiple signaling pathways and related genes. The polymorphism of a single minor gene might not be enough to cause obvious changes in serum SREBP2 protein expression. Moreover, the three tag SNPs examined in the present study are located in the intron region of the SREBP2 gene, and the polymorphism effects of SNPs in the intron region on gene expression are much less than those of SNPs in the promoter, 3 '-untranslated region, and open reading frame regions.

Lipid metabolism disorders have been recognized as a primary factor in ONFH pathogenesis. However, the association between SREBP2 polymorphism and the lipid metabolism disorder of ONFH has never been reported. Considering the 
A

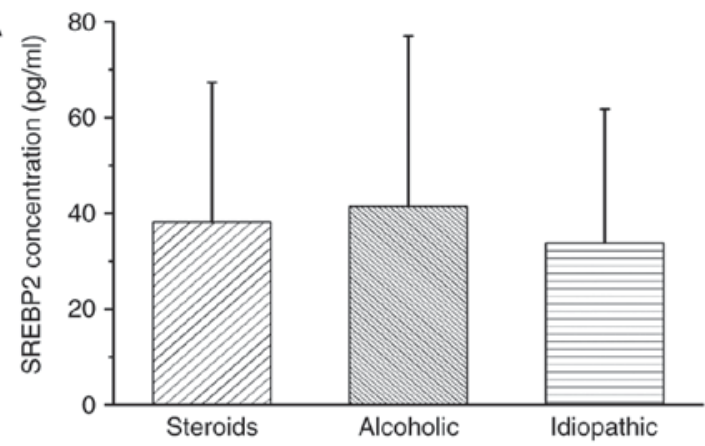

C
$\mathrm{B}$

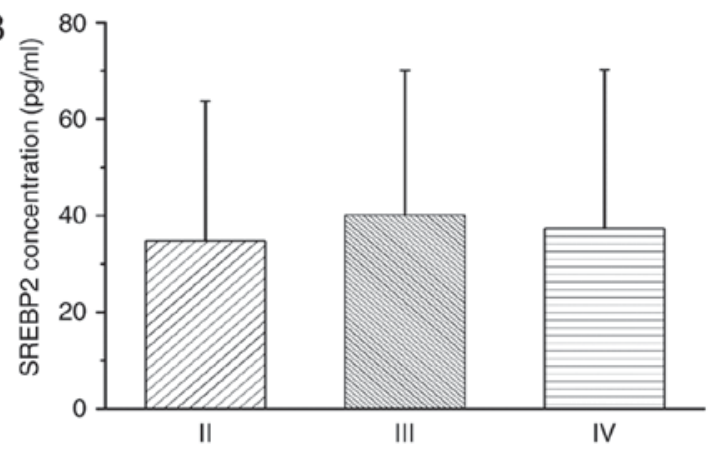

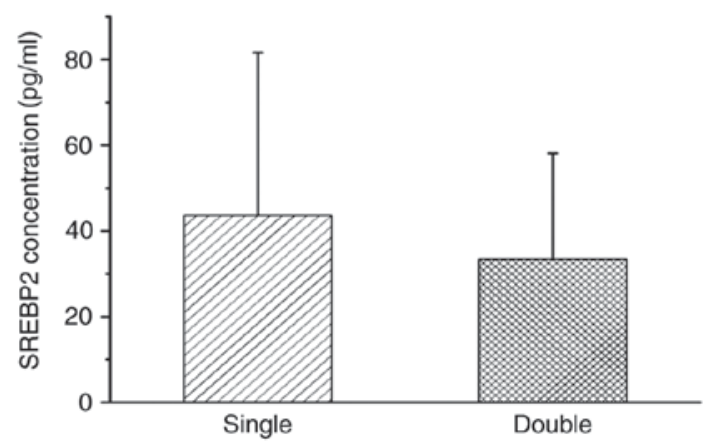

Figure 3. The association of protein expression of the SREBP2 gene with the clinical phenotypes of ONFH. (A) The association of the protein expression of the SREBP2 gene with the clinical etiological classification of ONFH. (B) The association of the protein expression of the SREBP2 gene with the clinical stages of ONFH. (C) The association of the protein expression of the SREBP2 gene with unilateral or bilateral hip lesions of ONFH. Data are presented as mean \pm standard deviation. SREBP2, sterol regulatory element-binding protein 2; ONFH, osteonecrosis of the femoral head.

important role of SREBP2 in the cholesterol synthesis and lipid metabolism, the authors first conducted the simultaneous detection of SREBP2 gene polymorphism and gene expression and ONFH lipid metabolism disorder. The results showed that ONFH lipid metabolism disorder was characterized by an increase in serum TG and LDL-c levels and the LDL-c/HDL-c ratio and a decrease in HDL-c levels. Furthermore, the serum LDL-c levels of rs2267443 GG or AG genotype carriers were significantly higher than those of AA genotype carriers, suggesting that SREBP2 polymorphism is associated with the lipid metabolism disorder of ONFH.

Excess glucocorticoids lead to an obvious increase of serum lipid levels and the necrosis of the femoral head (18). In an experiment animal model of ONFH induced by excess glucocorticoids, lipid droplets were observed in the bone cells concurrently with increased serum lipids (19). A study of $240 \mathrm{ONFH}$ patient-control systems showed that the serum adiponectin levels of ONFH patients were significantly lower than those of the healthy control subjects. Moreover, serum adiponectin levels positively associated with HDL-c levels and negatively associated with body mass index, TG levels, and the LDL/HDL ratio (20). Therefore, the serum adiponectin level was considered to be an independent predictor of ONFH risk. The pravastatin has been shown to prevent experimental ONFH induced by steroids, but the exact mechanism is unclear. A recent study using a rat $\mathrm{ONFH}$ model further demonstrated that pravastatin downregulated the expression of peroxisome proliferator activated receptor $\gamma$, a key transcription factors of adipogenic differentiation, and upregulated the expression of wingless-type MMTV integration site family $3 \mathrm{~A}$, LDL receptor related protein 5 , beta catenin and Runt-related transcription factor 2 genes on the signaling pathway of osteogenic differentiation at the mRNA and protein levels and prevented ONFH induction by steroids, providing the molecular evidence of lipid metabolism disorders affecting bone metabolism (21).

Bone marrow stromal cells (BMSCs) are a common precursor of osteoblasts and adipocytes. Using an in vivo diffusion chamber assay, preadipocytes from BMSCs enter an activated proliferation stage and differentiate into osteoblasts (22). The study using lithium chloride ( $\mathrm{LiCl})$ to reverse the abnormal adipogenic and osteoblastic differentiation of ONFH showed that LiCl significantly increased the expression of osteocalcin and Runx2 and enhanced osteogenic mineralization during the osteogenic induction process and also significantly reduced the formation of lipid droplets in the cells and the antagonism of adipogenesis by inhibiting PPAR $\gamma$ and fatty acid binding protein 4 expression in BMSCs during the induced adipogenic differentiation process (23). These results demonstrated that the lipid metabolism disorder serve critical roles in the development of ONFH. The present results further confirmed the lipid metabolism disorder of ONFH as well as the lipid metabolism disorder significantly associated with the SREBP2 genotypes, first suggesting the evidence SREBP2 gene polymorphisms intruding in the lipid metabolism disorder of ONFH. The complicated effects of SREBP2 on lipid homeostasis relate to the molecular functions of multiple signaling pathways. The detailed roles of SREBP2 gene in the lipid metabolism disorder of ONFH need to be elucidated.

In conclusion, the authors simultaneously showed the gene polymorphism and gene expression of SREBP2, as well as the lipid metabolism disorder in 182 ONFH patients and 179 healthy controls and their association with the 
clinical phenotypes and development of ONFH. The results demonstrated that SREBP2 gene polymorphisms are closely associated with lipid metabolism disorder and the development of ONFH.

\section{Acknowledgements}

The authors appreciate the support from all of the surgeons in the Department of Orthopedics who were involved in patient care and control and all of the graduate students of The Research Center who were involved in genomic DNA extraction. This work was supported by the Projects of Health Management Department of the Jilin Province, China (grant no. 20132003), the Project of Bethune Youth Foundation of Jilin University, China (grant no. 2015409), the Department of Science and Technology of the Jilin Province, China (grant nos. 20140311006YY and 20150312022ZG), and the Development and Reform Commission of the Jilin Province, China [grant no. 2014G073, the Project of Application Demonstration Center of Precision Medicine for Molecular Diagnosis in Jilin Province (2016-2018)].

\section{References}

1. Mont MA, Cherian JJ, Sierra RJ, Jones LC and Lieberman JR: Nontraumatic osteonecrosis of the femoral head: Where do we stand today? A ten-year update. J Bone Joint Surg Am 97: $1604-1627,2015$.

2. Moya-Angeler J, Gianakos AL, Villa JC, Ni A and Lane JM: Current concepts on osteonecrosis of the femoral head. World J Orthop 6: 590-601, 2015.

3. Chai W, Zhang Z, Ni M, Geng P, Lian Z, Zhang G, Shi LL and Chen J: Genetic association between methylenetetrahydrofolate reductase gene polymorphism and risk of osteonecrosis of the femoral head. Biomed Res Int 2015: 196495, 2015.

4. Wang Z, Zhang Y, Kong X, Li S, HU Y, Wang R, Li Y, Lu C, Lin $\mathrm{N}$ and Chen W: Association of a polymorphism in PON-1 gene with steroid-induced osteonecrosis of femoral head in Chinese Han population. Diagn Pathol 8: 186, 2013.

5. Kim H, Cho C, Cho Y, Cho S, Yoon K and Kim K: Significant associations of PAI-1 genetic polymorphisms with osteonecrosis of the femoral head. BMC Musculoskelet Disord 12: 160, 2011.

6. Peng KT, Huang KC, Huang TW, Lee YS, Hsu WH, Hsu RW, Ueng SW and Lee MS: Single nucleotide polymorphisms other than factor V Leiden are associated with coagulopathy and osteonecrosis of the femoral head in Chinese patients. PLoS One 9: e104461, 2014

7. Tang JJ, Li JG, Qi W, Qiu WW, Li PS, Li BL and Song BL: Inhibition of SREBP by a small molecule, betulin, improves hyperlipidemia and insulin resistance and reduces atherosclerotic plaques. Cell Metab 13: 44-56, 2011.
8. Bauer S, Wanninger J, Schmidhofer S, Weigert J, Neumeier M, Dorn C, Hellerbrand C, Zimara N, Schäffler A, Aslanidis C and BuechlerC: Sterol regulatory element-binding protein 2(SREBP2) activation after excess triglyceride storage induces chemerin in hypertrophic adipocytes. Endocrinology 152: 26-35, 2011.

9. Ficat RP: Idiopathic bone necrosis of the femoral head. Early diagnosis and treatment. J Bone Joint Surg Br 67: 3-9, 1985.

10. Singh DK, Rosenhouse-Dantsker A, Nichols CG, Enkvetchakul D and Levitan I: Direct regulation of prokaryotic kir channel by cholesterol. J Biol Chem 284: 30727-30736, 2009.

11. Boden G, Salehi S, Cheung P,Homko C, Song W, Loveland-Jones C and Jayarajan S: Comparison of in vivo effects of insulin on SREBP-1c activation and INSIG-1/2 in rat liver and human and rat adipose tissue. Obesity (Silver Spring) 21: 1208-1214, 2013.

12. Kostopoulou F, Gkretsi V, Malizos KN, Iliopoulos D, Oikonomou P, Poultsides L and Tsezou A: Central role of SREBP-2 in the pathogenesis of osteoarthritis. PLoS One 7: e35753, 2012.

13. Qiu XM, Jin CT and Wang W: Association between single nucleotide polymorphisms of sterol regulatory element binding protein-2 gene and risk of knee osteoarthritis in a Chinese Han population. J Int Med Res 42: 320-328, 2014.

14. Kim TH, Baek JI, Hong JM, Choi SJ, Lee HJ, Cho HJ, Park EK, Kim UK and Kim SY: Significant association of SREBP-2 genetic polymorphisms with avascular necrosis in the Korean population. BMC Med Genet 9: 94, 2008.

15. Song Y, Du ZW, Li QJ, Zhang GZ, Wang LL, Wu N, Wang JC and Gao ZL: Association of sterol regulatory element binding protein 2 and insulin-like growth factor binding protein 3 genetic polymorphisms with avascular necrosis of the femoral head in the Chinese population. Chin Med J (Engl) 125: 4037-4043, 2012.

16. Wang L, Luo DK and Pan ZY: Expression of 11 $\beta$-HSD in steroid-induced avascular necrosis of the femoral head. Mol Med Rep 7: 1482-1486, 2013.

17. Liu T, Su Q, Zang L, Kang N, Wang Y and Hai Y: Correlation between local microenvironment leptin expression and avascular necrosis of the femoral head. Zhongguo Xiu Fu Chong Jian Wai Ke Za Zhi 26: 1319-1323, 2012 (In Chinese).

18. Jones JP Jr: Fat embolism, intravascular coagulation, and osteonecrosis. Clin Orthop Relat Res: 294-308, 1993.

19. Tintut Y, Moronny S and demer LL: Hyperlipidemia promotes osteoclastic potential of bone marrow cells ex vivo. Arterioscler Thromb Vasc Biol 24: e6-e10, 2004.

20. Shuai B, Shen L, Yang YP, Xie J, Shou ZX and Wei B: Low plasma adiponectin as a potential biomarker for osteonecrosis of the femoral head. J Rheumatol 37: 2151-2155, 2010.

21. Jiang Y, Zhang Y, Zhang H, Zhu B, Li P, Lu C, Xu Y, Chen W and Lin N: Pravastatin prevents steroid-induced osteonecrosis in rats by suppressing PPAR $\gamma$ expression and activating Wnt signaling pathway. Exp Biol Med (Maywood) 239: 347-355, 2014.

22. Bennett JH, Joyner CJ, Triffitt JT and Owen ME: Adipocytic cells cultured from marrow have osteogenic potential. J Cell Sci 99: 131-139, 1991.

23. Yu Z, Fan L, Li J, Ge Z, Dang X and Wang K: Lithium prevents rat steroid-related osteonecrosis of the femoral head by $\beta$-catenin activation. Endocrine 52: 380-390, 2016. 